\title{
Development of a Risk Score for QT Prolongation in the Intensive Care Unit Using Time-Series Electrocardiogram Data and Electronic Medical Records
}

\author{
Tae Young Kim ${ }^{1}$, Byung Jin Choi ${ }^{1}$, Yeryung Koo ${ }^{1}$, Sukhoon Lee ${ }^{2}$, Dukyong Yoon ${ }^{1}$ \\ 'Department of Biomedical Informatics, Ajou University School of Medicine, Suwon, Korea \\ ${ }^{2}$ Department of Software Convergence Engineering, College of Industry-University Convergence Engineering, Kunsan National University, Gunsan, Korea
}

Objectives: Drug-induced QT prolongation can lead to life-threatening arrhythmia. In the intensive care unit (ICU), various drugs are administered concurrently, which can increase the risk of QT prolongation. However, no well-validated method to evaluate the risk of QT prolongation in real-world clinical practice has been established. We developed a risk scoring model to continuously evaluate the quantitative risk of QT prolongation in real-world clinical practice in the ICU. Methods: Continuous electrocardiogram (ECG) signals measured by patient monitoring devices and Electronic Medical Records data were collected for ICU patients. QT and RR intervals were measured from raw ECG data, and a corrected QT interval (QTc) was calculated by Bazett's formula. A case-crossover study design was adopted. A case was defined as an occurrence of QT prolongation $\geq 12$ hours after any previous QT prolongation. The patients served as their own controls. Conditional logistic regression was conducted to analyze prescription, surgical history, and laboratory test data. Based on the regression analysis, a QTc prolongation risk scoring model was established. Results: In total, 811 ICU patients who experienced QT prolongation were included in this study. Prescription information for 13 drugs was included in the risk scoring model. In the validation dataset, the high-risk group showed a higher rate of QT prolongation than the low-and low moderate-risk groups. Conclusions: Our proposed model may facilitate risk stratification for QT prolongation during ICU care as well as the selection of appropriate drugs to prevent QT prolongation.

Keywords: Cardiac Arrhythmias, Torsades de Pointes, Intensive Care Units, Electrocardiography, Risk Assessment

Submitted: October 28, 2020

Revised: December 19, 2020

Accepted: December 21, 2020

\section{Corresponding Author}

Dukyong Yoon

Department of Biomedical Informatics, Ajou University School of Medicine, 206 World cup-ro, Yeongtong-gu, Suwon 16499, Korea. Tel: +82-10-9300-5862, E-mail: d.yoon.ajou@gmail.com (https:// orcid.org/0000-0003-1635-8376)

This is an Open Access article distributed under the terms of the Creative Commons Attribution Non-Commercial License (http://creativecommons.org/licenses/by$\mathrm{nc} / 4.0 /$ ) which permits unrestricted non-commercial use, distribution, and reproduction in any medium, provided the original work is properly cited.

(C) 2021 The Korean Society of Medical Informatics

\section{Introduction}

QT prolongation is a common adverse event that can lead to various other adverse events. Approximately $24 \%$ of acutely ill patients experience QT prolongation and torsade de pointes, which is a complication of QT prolongation that is reportedly responsible for $6 \%$ of in-hospital cardiac arrests [1]. QT prolongation can increase the length of hospital stay and approximately triple mortality rate [1].

Patients admitted to the intensive care unit (ICU) are more likely to experience QT prolongation because they have more severe conditions, such as hypokalemia, hypomagnesemia, 
recent conversion from atrial fibrillation to sinus rhythm, or acute cerebral illness [2,3]. Moreover, these patients tend to take multiple drugs, including ones capable of prolonging the QT interval $[4,5]$ such as haloperidol, amiodarone, and furosemide. The risk of QT prolongation due to drugdrug interactions is also higher in these patients $[2,5,6]$. The prevalence of QT prolongation has been reported as $28 \%$ to $30 \%$ among ICU patients $[4,5]$. In one study, severe QT prolongation ( $>500 \mathrm{~ms}$ ) was observed in $18.2 \%$ of ICU patients, approximately half of whom were exposed to QT-prolonging drugs despite severe QT prolongation [6]. In other studies, it was estimated that approximately half of all ICU patients may develop QT prolongation $[7,8]$. QT prolongation is particularly common postoperatively (67\% to $80 \%)[9,10]$.

Although various scoring systems and risk evaluation models have been used for general in-hospital patients, there are no reliable models for evaluating QT prolongation risk in ICU patients. The RISQ-PATH score model was developed to identify patients who have a high risk of QT prolongation based on data from patients receiving haloperidol or an antimicrobial, and it has both high sensitivity and a high negative predictive value [11]. Other risk models to predict corrected QT interval (QTc) prolongation exhibit sensitivity, specificity, and area under the receiver operating characteristic curve (AUC) values of $0.77-0.84,0.28-0.48$, and $0.59-0.71$, respectively $[12,13]$. However, all these models were based on general in-hospital patients. The risk score introduced by Tisdale et al. [6] was developed for a cardiac critical care unit, but it exhibited low specificity when applied to the general ICU [14].

To overcome the current limitations of applying QT prolongation risk evaluation models in an ICU setting, we developed the "ICU-QT scoring system" based on time-series electrocardiogram (ECG) data collected from patient monitors installed in several ICUs. This system provides information on QT prolongation risk by analyzing real-time timeseries data, which could be useful for clinical monitoring and may inform drug administration decisions.

\section{Methods}

This study was approved by the Institutional Review Board of Ajou University Hospital (No. AJIRB-MED-MDB-19406). The requirement for informed consent was waived.

\section{Data Sources}

This study used data from the biosignal database and Electronic Medical Records of Ajou University Hospital, a tertia- ry teaching hospital in South Korea. The biosignal database contains ECG, arterial blood pressure, and photoplethysmogram data from patients admitted to the trauma ICU, medical ICU, emergency ICU, and neurological ICU [15]. The ECG waveforms used in this study were collected between September 2016 and May 2018. The ECG data obtained by Nihon Kohden and Philips patient monitors had sampling rates of 250 and $500 \mathrm{~Hz}$, respectively. Clinical information (demographics, prescription and diagnostic records, laboratory test results, and surgical histories) were obtained from patients' Electronic Medical Records during the same period.

\section{OTc Measurement and Target Event Definition}

Because the waveform data in the biosignal database only contain raw waveform signals, the onset of the QRS complex, R peak, and offset of the T-wave were detected using the QT detector developed by Chesnokov et al. [16] for the first 10-second segment of ECG data every hour. A QT interval was defined as the time between the onset of the QRS complex and the offset of the T-wave. An RR interval was defined as the time between successive $\mathrm{R}$ peaks. The median QT and RR intervals for 10-second segments were included in the analysis. QT intervals $\geq 800 \mathrm{~ms}$ were considered as outliers and excluded from the analysis. Based on the measured QT and RR intervals, QTc intervals were calculated (corrected for heart rate) using Bazett's formula:

$$
Q T c=\frac{Q T}{\sqrt{R R}}
$$

QT prolongation was defined as QTc (calculated using Bazett's formula) $>450 \mathrm{~ms}$ for men and $>470 \mathrm{~ms}$ for women.

\section{Development of the OT Prolongation Risk Evaluation Model for ICU Patients}

To develop the QT prolongation risk evaluation model, a case-crossover study design was used to minimize the effects of confounding variables, i.e., by using the patients as their own control group. Moreover, a longitudinal design was used so that the characteristics of the ECG data could be evaluated over time (Figure 1). A case was defined as an occurrence of QT prolongation $\geq 12$ hours after any previous QT prolongation. Controls referred to an absence of QT prolongation for $\geq 12$ hours. Cases and controls that overlapped within a 12-hour time window were excluded. Up to 16 cases and 16 controls were selected for each patient (derivation dataset). Levels of exposure to risk factors were compared between the periods before cases and controls (Figure 1).

Prescribed drugs, laboratory test results, and surgical histo- 


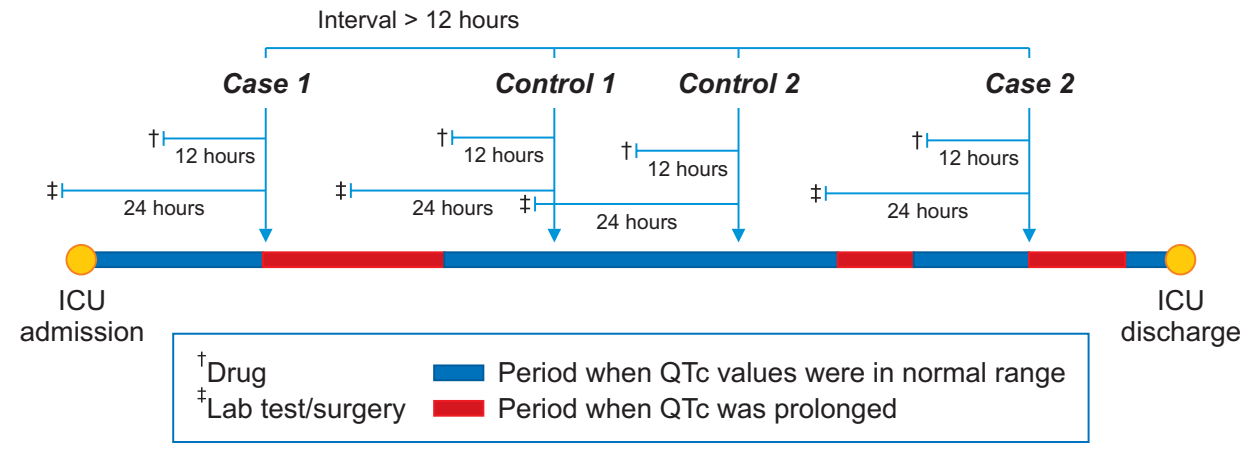

Figure 1. Schematic of the derivation of weights for risk factors in the OT prolongation risk scoring model. ICU: intensive care unit. ries were the potential risk factors for QT prolongation in the ICU patients in this study. The 300 drugs and 100 surgeries most frequently prescribed and performed, respectively, in these patients were analyzed. The observation windows for drugs and surgeries were set at 12 and 24 hours before each case and control, respectively. The serum potassium and calcium levels measured at the time closest to a drug case or control within $\leq 24$ hours before cases and controls were included in the analysis. Conditional logistic regression was performed to determine the risk posed by each factor, and $p$-values $<0.05$ were considered statistically significant. The case-crossover study design removes the bias associated with inter-individual differences. However, this approach does not evaluate the impact of patient characteristics.

To derive the ICU-QT score, beta coefficient weights were used; these were multiplied by 10 and rounded to the nearest integer. The ICU-QT score was defined as

$$
\text { ICU-QT score }=\sum(w \times x)
$$

where $w$ represents the weight value, and $x$ represents the value of each variable. This approach yielded a superior net reclassification index compared with various other approaches [17].

\section{Performance Evaluation}

Performance evaluation was conducted in three steps. First, we checked for consistency with previous studies in terms of the factors showing significant associations with QT prolongation. Second, the incidence of QT prolongation was analyzed by ICU-QT score in both the derivation and validation datasets. The validation dataset consisted of data for different time points from the same patients used for model development. Finally, low-, low moderate-, high moderateand high-risk QT prolongation groups were distinguished based on the chi-squared test and pairwise Fisher exact test with Bonferroni correction.
Table 1. Baseline characteristics of the patients in this study

\begin{tabular}{lc}
\hline \multicolumn{1}{c}{ Characteristic } & Value \\
\hline Total number of patients & $811(100)$ \\
Sex & \\
Male & $560(69.1)$ \\
Female & $251(30.9)$ \\
Age (yr) & $54.5 \pm 20.4$ \\
Type of ICU & \\
Trauma & $507(62.5)$ \\
Medical & $123(15.2)$ \\
Emergency & $155(19.1)$ \\
Neurological & $26(3.2)$ \\
Number of QT prolongations per patient & $4.39 \pm 4.00$ \\
\hline
\end{tabular}

Values are presented as number (\%) or mean \pm standard deviation.

ICU: intensive care unit.

\section{Software}

Data were managed using Microsoft SQL Server and Python; R software (R Development Core Team, Vienna, Austria) was used for the statistical analyses.

\section{Results}

In total, $811 \mathrm{ICU}$ patients were included in this study (560 men); 507 patients were admitted to the trauma ICU (Table 1). The mean number of QT prolongations per patient during the ICU stay was 4.39 .

Drugs significantly associated with QT prolongation in this study are shown in Table 2. Changes in laboratory test results (i.e., serum potassium and calcium levels) and surgical history were not significantly associated with QT prolongation, whereas 13 drugs did show a significant association. There was previous evidence of five of those drugs being associated with QT prolongation.

With increasing ICU-QT score, the rate of QT prolongation 
Table 2. Drugs significantly associated with OT prolongation and weights derived for the development of the ICU-QT scoring model

\begin{tabular}{lcclcr}
\hline \multicolumn{1}{c}{ Drug } & $\beta$ & $p$-value & Known risk factor & Accordance with previous data & Weight \\
\hline Isoconazole nitrate & -1.454 & 0.003 & No evidence & $\triangle$ & -15 \\
Lorazepam & 0.402 & 0.033 & No evidence & $\triangle$ & 4 \\
Aspirin & 0.474 & 0.040 & No evidence & $\triangle$ & 5 \\
Imipenem/cilastatin & -0.642 & 0.019 & No evidence & $\triangle$ & -6 \\
Budesonide & -0.674 & 0.012 & No evidence & $\triangle$ & -7 \\
Nicardipine & 0.593 & $<0.001$ & Increase & $\mathrm{O}$ & 6 \\
Dexmedetomidine & -0.488 & $<0.001$ & Increase & a) decrease & -5 \\
Lafutidine & 0.573 & 0.020 & No evidence & $\mathrm{O}$ & 6 \\
Levetiracetam & 0.376 & 0.017 & Increase & $\triangle$ & 4 \\
Rocuronium & 0.746 & 0.012 & Increase & $\mathrm{O}$ & 7 \\
Norepinephrine & 0.382 & 0.014 & Increase & $\mathrm{O}$ & 4 \\
Vecuronium & 0.415 & 0.030 & No evidence & $\mathrm{O}$ & 4 \\
Propacetamol & 0.290 & 0.012 & No evidence & $\triangle$ & 3 \\
\hline
\end{tabular}

ICU: intensive care unit.

${ }^{\text {a) }}$ From Woosley et al. [21].

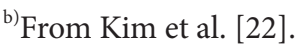

${ }^{c)}$ From Agdanli et al. [23].

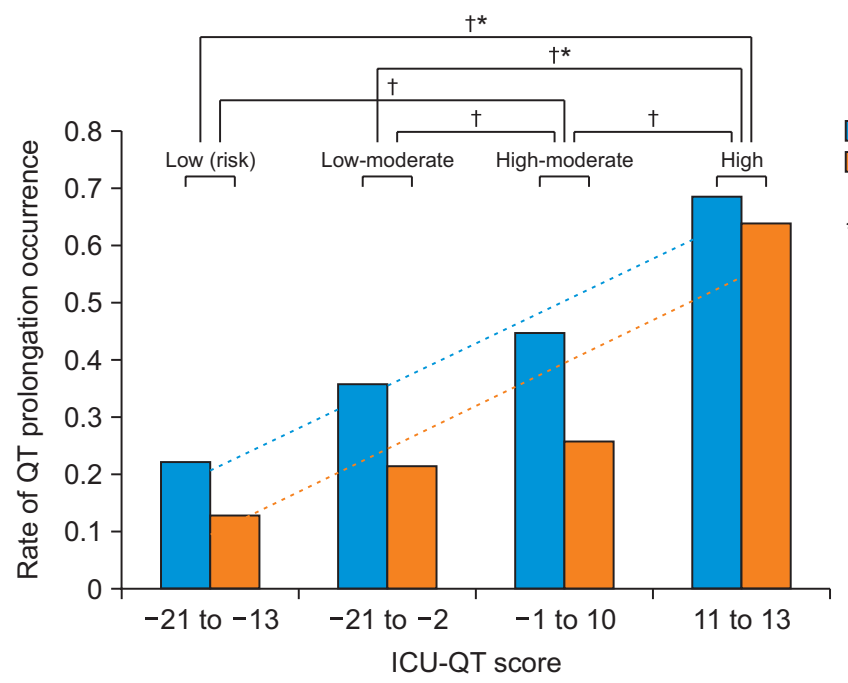

increased, as shown in Figure 2, in both the derivation and validation datasets. The rate of QT prolongation increased by approximately $7 \%$ for each 5-point increase in ICU-QT score (6.6\% in the derivation dataset and $7.3 \%$ in the validation dataset).

Rates of QT prolongation differed significantly among the low-, low moderate-, high moderate- and high-risk groups in the derivation $(p<0.001)$ and validation datasets $(p=$ $0.007)$. In the post hoc analysis, there were significant differences between the low- and high-risk groups in both the derivation $(p<0.001)$ and validation datasets $(p=0.02)$ and
Derivation dataset

Validation dataset

${ }^{\dagger} p<0.05$ in derivation dataset

${ }^{*} p<0.05$ in validation dataset

Figure 2. Rate of QT prolongation according to ICU-QT score. ICU: intensive care unit. 
Table 3. OT prolongation count in the validation dataset by risk group

\begin{tabular}{lcc}
\hline & OT prolongation & Normal \\
\hline Low interval \\
\hline Low-moderate risk & 3 & 21 \\
High-moderate risk & 59 & 218 \\
High risk & 725 & 2,136 \\
\hline
\end{tabular}

\section{Discussion}

We developed the ICU-QT score to provide information regarding the risk of QT prolongation in ICU patients. In both the derivation and validation datasets, the ICU-QT score was strongly correlated with the risk of QT prolongation in real-world ICU settings.

Although the risk of QT prolongation is greater in ICU patients, most previous models evaluating the risk of QT prolongation were developed using data from non-ICU patients. ICU patients usually have more severe clinical conditions and a higher likelihood of exposure to multiple drugs or injections. Notably, disease severity differs between patients in the ICU and general wards, while taking multiple drugs can increase the risk of drug-drug interactions, potentially resulting in QT prolongation $[2,5,6]$.

Using ECG data collected from ICU patients [18], we developed a QT prolongation risk evaluation model. A longitudinal case-crossover study design was used to remove the bias associated with inter-individual differences.

Five of the 13 drugs (nicardipine, dexmedetomidine, levetiracetam, rocuronium, and norepinephrine) showing significant associations with QT prolongation in this study were also significantly associated with QT prolongation in previous studies. The other five drugs (lorazepam, aspirin, lafutidine, vecuronium, and propacetamol) were newly identified to have significant adverse associations with QT prolongation in ICU patients. Because most studies have focused on drugs that can lead to QT prolongation, there are insufficient data regarding drugs that have no relationship or a negative relationship (i.e., a protective effect). In this study, isoconazole nitrate, imipenem/cilastatin, budesonide, and dexmedetomidine were found to have protective effects.

Our scoring model is intended to quantify the overall risk of QT prolongation rather than to obtain the odds ratios for individual risk factors. Therefore, caution is needed when interpreting the results. The variables included in this study were used as surrogate markers of clinical status, and were weighted according to the risk of QT prolongation in real- world clinical practice. For example, the drug vecuronium is typically used in patients receiving mechanical ventilator care, and it prevents resistance against the ventilator by relaxing the skeletal muscles. In this case, the weight of vecuronium can be interpreted as the QT prolongation risk posed by severe clinical conditions that require ventilator care or the risk associated with ventilator use itself.

This study had the following limitations. First, data were collected from a single institute and primarily from the trauma ICU (62.5\% of cases and controls) because our "biosignal collecting system" was initially installed in the trauma ICU before later being implemented in other ICUs. In a future study, we will use a more balanced ICU dataset, by collecting additional data from other ICUs, and we will perform model validation using an external dataset. Second, as previously mentioned, our results do not allow causality to be inferred regarding the relationships between risk factors and QT prolongation. To develop a more precise model, detailed clinical information (e.g., on medical device usage, procedures conducted during ICU stays, and vital signs) would be helpful. However, such information might be stored in different data formats across hospitals, so the added complexity could hinder adoption of the model in clinical practice. Therefore, we only used prescription information, which is usually stored in a simple format and can well represent a variety of clinical situations. Third, the variety of half-lives of drugs was not considered. Some drugs, benzodiazepine like lorazepam could have an effect for more than 12 hours, which was set as the observation time window in this study. However, to increase the usability of the users and with the assumption that drugs administered near an event could have more significant effects, we simplified the observation time window as 12 hours.

Some studies have reported that a clinical decision support system has proven effective to reduce adverse events caused by QT prolongation $[19,20]$. Therefore, we expect that models like ours can help to reduce the clinical deterioration of patients in ICU caused by QT prolongation. To realize this aim, research to improve QT prolongation event detection must be continued.

In conclusion, we developed the ICU-QT scoring system that can support clinicians in assessing the risk of QT prolongation in real time. It can facilitate QT control and reduce the use of QT-prolonging drugs in patients at high risk of QT prolongation. 


\section{Conflict of Interest}

No potential conflict of interest relevant to this article was reported.

\section{Acknowledgments}

This research was supported by a grant (No. 19182MFDS406) from Ministry of Food and Drug Safety in 2020. This research was also supported by a Government-wide R\&D Fund project for infectious disease research (GFID), Republic of Korea (No. HG18C0067).

\section{ORCID}

Tae Young Kim (http://orcid.org/0000-0002-2591-0129) Byung Jin Choi (http://orcid.org/0000-0002-1445-5888) Yeryung Koo (http://orcid.org/0000-0002-6885-7663) Sukhoon Lee (http://orcid.org/0000-0002-3390-5602) Dukyong Yoon (http://orcid.org/0000-0003-1635-8376)

\section{References}

1. Pickham D, Helfenbein E, Shinn JA, Chan G, Funk M, Weinacker A, et al. High prevalence of corrected QT interval prolongation in acutely ill patients is associated with mortality: results of the QT in Practice (QTIP) Study. Crit Care Med 2012;40:394-9.

2. Etchegoyen CV, Keller GA, Mrad S, Cheng S, Di Girolamo G. Drug-induced QT interval prolongation in the intensive care unit. Curr Clin Pharmacol 2017;12:21022.

3. Beitland S, Platou ES, Sunde K. Drug-induced long QT syndrome and fatal arrhythmias in the intensive care unit. Acta Anaesthesiol Scand 2014;58:266-72.

4. George TK, Chase D, Peter JV, Satyendra S, Kavitha R, George LR, et al. Association between a prolonged corrected QT interval and outcomes in patients in a medical Intensive Care Unit. Indian J Crit Care Med 2015;19: 326-32.

5. Fernandes FM, Silva EP, Martins RR, Oliveira AG. QTc interval prolongation in critically ill patients: prevalence, risk factors and associated medications. PLoS One 2018;13:e0199028.

6. Tisdale JE, Wroblewski HA, Overholser BR, Kingery JR, Trujillo TN, Kovacs RJ. Prevalence of QT interval prolongation in patients admitted to cardiac care units and frequency of subsequent administration of QT interval- prolonging drugs: a prospective, observational study in a large urban academic medical center in the US. Drug Saf 2012;35:459-70.

7. Kozik TM, Wung SF. Acquired long QT syndrome: frequency, onset, and risk factors in intensive care patients. Crit Care Nurse 2012;32:32-41.

8. Hoogstraaten E, Rijkenberg S, van der Voort PH. Corrected QT-interval prolongation and variability in intensive care patients. J Crit Care 2014;29:835-9.

9. Nagele P, Pal S, Brown F, Blood J, Miller JP, Johnston J. Postoperative QT interval prolongation in patients undergoing noncardiac surgery under general anesthesia. Anesthesiology 2012;117:321-8.

10. Pham JC, Banks MC, Narotsky DL, Dorman T, Winters $\mathrm{BD}$. The prevalence of long QT interval in post-operative intensive care unit patients. J Clin Monit Comput 2016;30:437-43.

11. Vandael E, Vandenberk B, Vandenberghe J, Spriet I, Willems R, Foulon V. Development of a risk score for QTc-prolongation: the RISQ-PATH study. Int J Clin Pharm 2017;39:424-32

12. Bindraban AN, Rolvink J, Berger FA, van den Bemt PM, Kuijper AF, van der Hoeven RT, et al. Development of a risk model for predicting QTc interval prolongation in patients using QTc-prolonging drugs. Int J Clin Pharm 2018;40:1372-9.

13. Berger FA, van der Sijs H, Becker ML, van Gelder T, van den Bemt PM. Development and validation of a tool to assess the risk of QT drug-drug interactions in clinical practice. BMC Med Inform Decis Mak 2020;20:171.

14. Su K, McGloin R, Gellatly RM. Predictive validity of a QTc interval prolongation risk score in the intensive care unit. Pharmacotherapy 2020;40:492-9.

15. Chung D, Choi J, Jang JH, Kim TY, Byun J, Park H, et al. Construction of an electrocardiogram database including 12 lead waveforms. Healthc Inform Res 2018;24:2426.

16. Chesnokov YC, Nerukh D, Glen RC. Individually adaptable automatic QT detector. Proceedings of 2006 Computers in Cardiology; 2016 Sep 17-20; Valencia, Spain, p. $337-40$

17. Mehta HB, Mehta V, Girman CJ, Adhikari D, Johnson ML. Regression coefficient-based scoring system should be used to assign weights to the risk index. J Clin Epidemiol 2016;79:22-8.

18. Yoon D, Lee S, Kim TY, Ko J, Chung WY, Park RW. System for collecting biosignal data from multiple patient monitoring systems. Healthc Inform Res 2017;23:333-7. 
19. Sharma S, Martijn Bos J, Tarrell RF, Simon GJ, Morlan BW, Ackerman MJ, et al. Providers' response to clinical decision support for QT prolonging drugs. J Med Syst 2017;41:161.

20. Tisdale JE, Jaynes HA, Kingery JR, Overholser BR, Mourad NA, Trujillo TN, et al. Effectiveness of a clinical decision support system for reducing the risk of QT interval prolongation in hospitalized patients. Circ Cardiovasc Qual Outcomes 2014;7:381-90.

21. Woosley RL, Heise CW, Gallo T, Tate J, Woosley D, Romero KA. QTdrugs list [Internet]. Oro Valley (AZ): AZCERT Inc.; c2013 [cited 2021 Mar 30]. Available from: https://www.crediblemeds.org/.

22. Kim NY, Han DW, Koh JC, Rha KH, Hong JH, Park JM, et al. Effect of dexmedetomidine on heart rate-corrected QT and Tpeak-Tend intervals during robot-assisted laparoscopic prostatectomy with steep Trendelenburg position: a prospective, randomized, double-blinded, controlled study. Medicine (Baltimore) 2016;95:e3645.

23. Agdanli D, Ozturk T, Utuk O, Keles GT. Effects of highdose rocuronium on the QTc interval during anaesthesia induction in patients undergoing coronary artery bypass graft surgery. Turk J Anaesthesiol Reanim 2014;42:24550. 